\title{
Comparison of Pain Measures Between Tendons of Elite Basketball Players With Different Sonographic Patterns
}

\author{
Josep C. Benítez-Martínez, Pablo Martínez-Ramírez, Fermín Valera-Garrido, \\ Jose Casaña-Granell, and Francesc Medina-Mirapeix
}

\begin{abstract}
Context: The prevalence and negative consequences of the symptoms surrounding patellar tendinopathy constitute an important problem for sports medicine professionals. The identification of potential pain mediators is, therefore, of major interest to improve both the prevention and management of this injury. Objective: To compare the pain experienced by elite male adult basketball players and patterns of patellar tendon abnormalities. Also, to identify whether structural and vascular sonographic abnormalities (focal area of hypoechogenicity, thickening, and neovascularization [NV]) are equal in determining pain perceptions. Design: An observational study with professional basketball teams (ACB-Spanish league). Participants: A total of 73 male basketball players (mean age 26.8 y). Main Outcome Measures: Patellar tendon ultrasonography images. Pain scores were compared between the identified patterns. Multiple regression analysis was used to examine the relative importance of abnormalities. Results: Of the 146 tendons, 91 had some degree of sonographic abnormality. Three main patterns were identified: I (1 structural abnormality without NV), II ( 2 structural abnormalities without NV), and III ( 2 structural abnormalities and NV). A total of 31 tendons (21.2\%) exhibited pattern I, $46(31.5 \%)$ presented pattern II, and 13 tendons (8.9\%) exhibited pattern III. The mean visual analog scale and the Victorian Institute of Sport assessment questionnaire-patellar tendon (VISA-P) scores for pattern III were significantly different $(P<.05)$ compared with patterns I and II; however, the pain pressure threshold (PPT) scores were not. NV was significantly associated with worsened scores for all pain measures; however, the focal area of hypoechogenicity was only associated with PPT scores. Conclusion: Patterns of sonographic abnormalities, including NV, demonstrated greater pain. Although NV determined scores for the visual analog scale, VISA-P, and PPT, the presence of focal area of hypoechogenicity on its own is a determining factor for the PPT. This study suggests that the combination of 2 or more sonographic abnormalities may help explain pain variations among basketball players.
\end{abstract}

Keywords: tendon injury, ultrasonography, patellar tendinopathy, hypoechogenicity, tendon symptoms

Patellar tendinopathy (PT) is a chronic overuse injury prevalent among jumping athletes, such as elite and nonelite basketball and volleyball players. ${ }^{1,2}$ Pain associated with PT can limit an athlete's ability to jump or run and negatively affecting training or competition levels. ${ }^{3,4}$ Given the prevalence and negative consequences of the symptoms surrounding PT, research aimed at identifying potential pain mediators is of major interest to improve both the prevention and management of this injury.

Abnormal intratendinous structural changes, such as hypoechoic areas or thickening (Thk) and abnormal vascular structures (neovascularization $[\mathrm{NV}]$ ), detected with Doppler ultrasound, have been shown to be an important source of patellar tendon pain. ${ }^{5-7}$ In cases of tendinopathy, several histological findings have been suggested to be the cause of the changes observed in the pathology of the tendon; these are the irregular structure of the fiber and the high concentration of glycosaminoglycans and vascular ingrowth. ${ }^{8}$ Furthermore, these findings may explain changes detected by ultrasound, such as hypoechoic areas and increased thickness. Alfredson et $\mathrm{al}^{9}$ supported neovessels and

Benítez-Martínez and Casaña-Granell are with the Department of Physical Therapy, University of Valencia, Valencia, Spain. Martínez-Ramírez is with ACB team, Valencia Basket Club, Valencia, Spain. Valera-Garrido is with MVClinic, Madrid, Spain; and has a Master's degree in Invasive Physiotherapy Techniques, CEU San Pablo University, Madrid, Spain. Medina-Mirapeix is with the Department of Physical Therapy, University of Murcia, Murcia, Spain. Benitez-Martinez (josep. benitez@uv.es) is corresponding author. accompanying nerves being the possible source of pain in chronic midportion Achilles tendinosis. Nevertheless, the clinical relevance of these abnormalities is a matter of debate, because these have also been reported to be present in asymptomatic individuals. ${ }^{5,10}$ Some relevant research regarding this debate has found that the combination of 2 or more sonographic abnormalities rather than the evaluation of isolated characteristics may offer some clarification regarding pain variations. ${ }^{11,12}$ Along these lines, Cook et $\mathrm{al}^{11}$ demonstrated that the presence of NV in abnormal patellar tendons with structural changes among a group of volleyball players was associated with greater tendon pain when compared with abnormal tendons without NV. Despite the relevance of the combination of vascular and structural abnormalities for explaining pain variations, there are few studies comparing pain in patellar tendons with different combinations of sonographic abnormalities (eg, 2 intratendinous structural changes vs only 1 ).

This study investigated the patellar tendons of active elite basketball players to assess the status of their tendons and establish patterns based on the combination of the presence/absence of NV and 2 sonographic structural abnormalities: focal area of hypoechogenicity (FAH) and Thk. The aim of this study was to compare pain associated with different patterns of abnormal tendons present in a cohort of elite basketball players. A secondary aim of this study was to identify whether structural and vascular sonographic abnormalities are equal in determining different pain measures in the patellar tendon among elite players. 


\section{Methods}

\section{Study Design and Participants}

This was an observational study performed on male professional basketball players in the first division of the Spanish league. This study was conducted according to the regulations of the University of Valencia Ethical Committee for Human Research and approved with number H1456389710571. This study took place during the 2015/2016 basketball season, from November to May. All teams in the ACB (Basketball Clubs Association-professional Spanish league) were invited to participate. A total of 8 out of an estimated 16 teams teams agreed to participate. Players were initially recruited by their physiotherapists with their coach's knowledge and consent. The inclusion criteria were practicing the sport at the time of assessment, professional player, aged 18 or older. The exclusion criteria were systemic illness, history of knee surgery, receiving invasive therapy in the previous 3 months, analgesic or antiinflammatory drugs in the last 24 hours, and an inability to communicate in either Spanish or English. All eligible players were informed of the study purposes and their voluntary participation was requested. In addition, all participants signed an informed consent form.

\section{Measures}

All data were collected by 2 experienced sports physiotherapists using a standardized procedure during a session which took place at the end of the week, between 12 and 24 hours prior to the regular match. One professional collected demographic (age), anthropometric (height and weight), and questionnaire-based pain measures of the patellar tendon for both the dominant and nondominant side. A second professional (PMR), who was blinded to the questionnairebased pain scores, assessed the pain pressure threshold (PPT) and status of both tendons using an ultrasound protocol.

\section{Pain Measures}

Three separate measures were used to record different aspects of patellar tendon pain: a visual analog scale (VAS), the Victorian Institute of Sport assessment questionnaire-patellar tendon (VISA-P), and the PPT. First, players were asked to complete a VAS to record maximal anterior knee pain during practice during the previous week, as described elsewhere. ${ }^{9}$ The VAS was a 100-mm long continuous line with a "no pain" descriptor on the left side and "worst pain" descriptor on the right.

The VISA-P questionnaire is a useful tool for identifying clinical severity, measuring symptoms, function, and sports participation in relation to PT. ${ }^{13}$ The VISA-P contains 8 questions about PT. The maximum score for an asymptomatic individual is 100 points. For the Spanish players, we used the Spanish version of the VISA-P, ${ }^{14}$ whereas an English version was used for the remaining players.

The PPT of players' patellar tendons was measured using an algometer (Pain Test-Model FPK 40; Wagner Instruments, Greenwich, CT). The device was applied to the tendon via a rubber disk with a surface measuring $1 \mathrm{~cm}^{2}$. The pressure applied (measured in kilograms) exhibits the tenderness with palpation of the tendon and it shows lower scores when the tendon is more affected and painful. Algometry was applied by the same examiner in all players without knowledge of VISA-P or VAS scores. The measurement of PPT was performed with players in a standardized supine position, with slightly flexed knees supported by a cushion under the popliteal fossa. The algometer was positioned directly distal to the patellar apex on the proximal side of the patellar tendon (ie, where the player experienced the most tenderness or pain). The patella was held in place by pressing upon the proximal pole of the patella. The algometer was pressed against the tenderest area of the patellar tendon, and the pressure was applied at $0.1 \mathrm{~kg} / \mathrm{s}$ increments until the patient experienced pain. This limit was set to avoid any harm to the knee. If players experienced any sense of pain, they were told to say "stop," at which point the algometer was immediately removed. The peak measurement force was displayed on the algometer and the means of 2 measurements were taken for analysis. The second measurement was taken immediately after the first one (with a minimum of $60 \mathrm{~s}$ between each measurement) and on the same spot. We compared the PPT and covariant data of players with and without PT.

\section{Ultrasound Protocol}

The patellar tendons of both knees were assessed with gray scale and Doppler color with a Sonosite ${ }^{\circledR}$ M-Turbo portable ultrasound device (Sonosite, Inc, Bothell, WA) equipped with a 10 - to $5-\mathrm{MHz}$ transducer head.

The patellar tendon examination protocol was based on the Musculoskeletal Ultrasound Technical Guidelines: Knee, defined by the European Society of Musculoskeletal Radiology. ${ }^{15}$ The subject was in a supine position with approximately $30^{\circ}$ knee flexion, obtained by placing a small pillow beneath the popliteal space. The tendons visualized in both longitudinal and transverse scans were recorded at the widest thickened point in the sagittal plane and at points at which abnormalities were observed. The axial image was taken $5 \mathrm{~mm}$ distal to the inferior pole of the patella. Subsequently, the knee was extended and the quadriceps muscles were relaxed to prevent physical constriction of the blood vessels. Power Doppler settings were standardized with an automatic gain, a sensitivity of 4 to $-4 \mathrm{~cm} / \mathrm{s}$, and $608-\mathrm{Hz}$ pulse repetition frequency. Power Doppler sonography was used to study blood flow in the patellar tendon. Because only high flows can be registered, this technique does not allow for registration of the normal circulation in the tendon, due to the relatively low flow rate.

The images were analyzed by 2 experienced musculoskeletal sonographers (PMR and FVG), first alone, and subsequently by consensus, in the event of any disagreements. The initial Kappa coefficient ranged from 0.88 to 0.99 for the 3 sonographic abnormalities (FAH 0.97, 95\% CI, 0.93-1; Thk 0.88, 95\% CI, 0.8-0.96; NV $0.99,95 \%$ CI, 0.98-1). FAH was considered present when a zone with focal changes in echogenicity was observed. Thk was considered present when a thickened tendon was observed in a particular site, altering the tendon symmetry in the transverse section. NV was considered present when the vessel shown within the tendon in the sagittal plane was demonstrated to be greater than $1 \mathrm{~mm}$ in length according to Doppler sonography assessment (Figure 1). Tendons that contained a focal hypoechoic region and diffuse Thk were categorized as focally hypoechoic.

\section{Statistical Analysis}

The number of sonographic abnormalities per tendon was calculated, and patterns were identified from all combinations of structural and vascular abnormalities present in each tendon.

Descriptive statistics were calculated, including frequency and proportions for categorical variables, and means and SDs for continuous variables. A 1-way between-groups analysis of variance was conducted to explore the impact of patterns of combined 


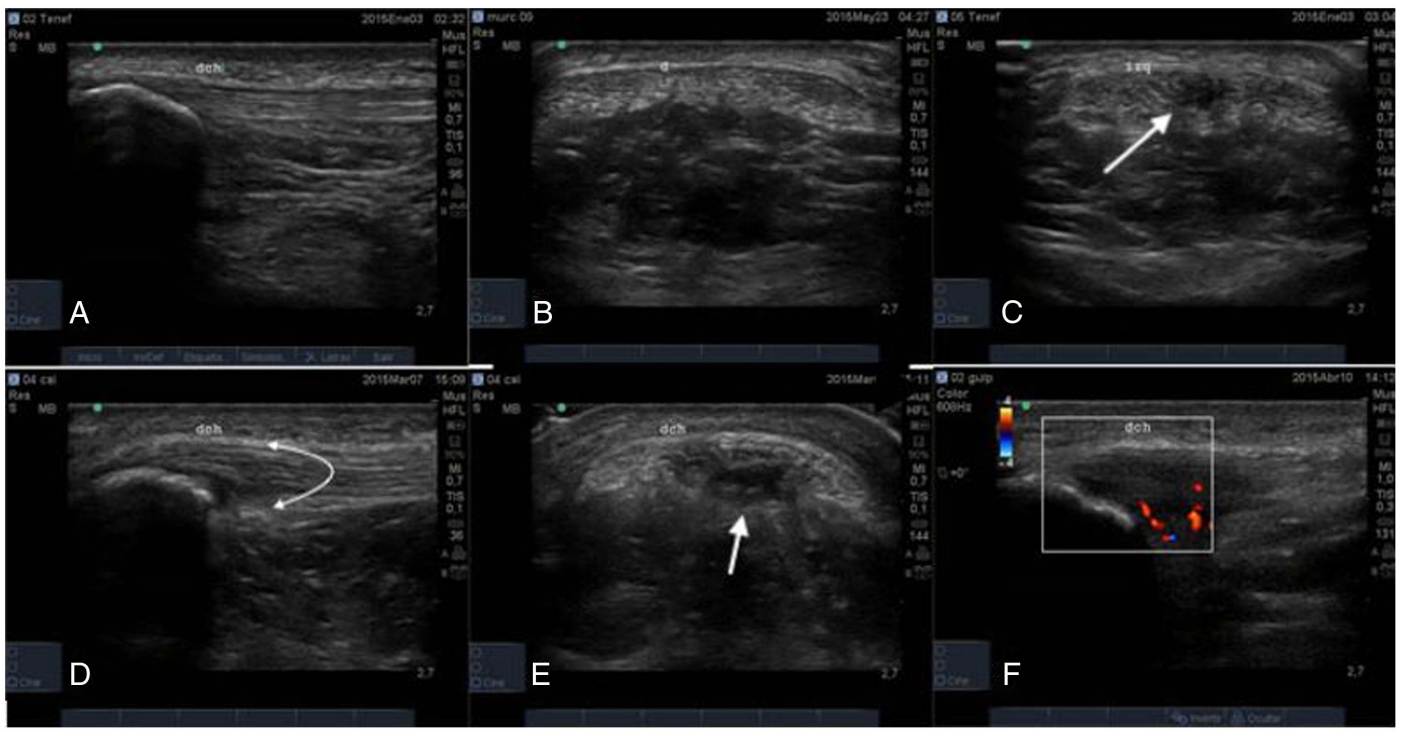

Figure 1 - Sonographic images of tendon assessments. Longitudinal (A) and transverse (B) scan of normal tendon; (C) tendon with FAH (white arrow) in transverse scan (pattern I); (D) tendon with FAH and thickening (white curved arrow), longitudinal scan (pattern II); (E) tendon with FAH (white arrow) and thickening, transverse scan (pattern II); (F) tendon with FAH, thickening, and neovascularization (pattern III; white box). FAH indicates focal area of hypoechogenicity.

abnormalities on the 3 pain measures (VAS, VISA-P, and PPT). Post hoc comparisons using the Bonferroni and Tukey tests were used to analyze differences between pairs of patterns. Multiple linear regression analyses were performed using the backward method to examine the relative contributions of the 3 sonographic abnormalities (FAH, Thk, and NV) for each of the 3 pain measures (VAS, VISA-P, and PPT).

\section{Results}

A total of 73 active players were included in the study, and 146 tendons were analyzed. The mean age of the subjects was 26.8 years $(\mathrm{SD}=4.8)$, the mean height was $198.1 \mathrm{~cm}(\mathrm{SD}=8.4)$, and the mean weight was $95.5 \mathrm{~kg}(\mathrm{SD}=11.4)$. Regarding the 146 tendons, the mean VAS was $0.8(\mathrm{SD}=1.7)$, the mean VISA-P was $93.6(\mathrm{SD}=$ $10.4)$, and the mean PPT was $10.3(\mathrm{SD}=2.7)$. The mean number of years in elite competition was 8.8 years $(\mathrm{SD}=4.8)$. Of the 146 tendons, 55 had no detectable tendon changes and 91 had abnormalities. Among the 73 players, $21(28.7 \%)$ subjects had unilateral tendon abnormalities, 35 (48\%) had bilateral abnormalities, and only $17(23.3 \%)$ had no abnormalities.

\section{Patterns Observed}

Among the 146 tendons, 91 were abnormal tendons, 90 (61.6\% of total) exhibited FAH, 59 (40.4\% of total) exhibited Thk, and 14 (9.6\% of total) exhibited NV. Figure 2 shows the 4 pattern combinations exhibited by the 91 tendons with abnormalities: $13(8.9 \%)$ presented pattern III ( 2 structural abnormalities and NV), $46(31.5 \%)$ showed pattern II (2 structural abnormalities without NV), 31 (21.2\%) displayed pattern I (only 1 structural abnormality without NV), and $1(0.6 \%)$ presented pattern I-b (only 1 structural abnormality, although with NV). Because this last pattern was very scarce, it was not included in the analyses.

\section{Pain Scores and Pattern}

Table 1 compares VAS, VISA-P, and PPT pain scores between patterns of abnormal and normal tendons. There was a statistically significant difference in VAS scores $\left(F_{3.142}=8.6, P<.001\right)$, VISA-P $\left(F_{3.142}=6.2, P=.001\right)$, and PPT scores $\left(F_{3.142}=4.8, P=.003\right)$ for the 4 groups. Post hoc comparisons indicated that the VAS and VISA-P mean scores for pattern III were significantly different from those for Patterns II and I and normal tendons. By contrast, post hoc comparisons indicated that the PPT mean score for pattern III was only significantly different from normal tendons. For all 3 pain measures, pattern I did not differ significantly from either pattern II or normal tendons.

Table 2 indicates the results of the multivariate sonographic determinants of each of the 3 pain measures. The presence of $\mathrm{NV}$ was significantly associated with worsened scores in all pain measures. By contrast, the presence of FAH was only associated with worsened PPT scores. Tendon Thk was not associated with worsened VAS, VISA-P, or PPT scores.

\section{Discussion}

To our knowledge, this is the first study to identify patterns of vascular and structural sonographic abnormalities in the patellar tendons of elite basketball players and to compare different aspects of pain among these patterns. Our study identified 3 main patterns or combinations of abnormalities. Pattern II (which combined FAH and Thk) was the most prevalent, and pattern III (FAH, Thk, and $\mathrm{NV}$ ) was the least common among the elite basketball players. This last pattern, the only one with NV, was the pattern that obtained the worst scores on the 3 pain measures used in this study. Consistent with this, NV was the only common determinant for the different pain measures for the patellar tendon in elite players. There was 1 tendon with NV that only had 1 structural abnormality. There was no reasonable explanation for this exception. 


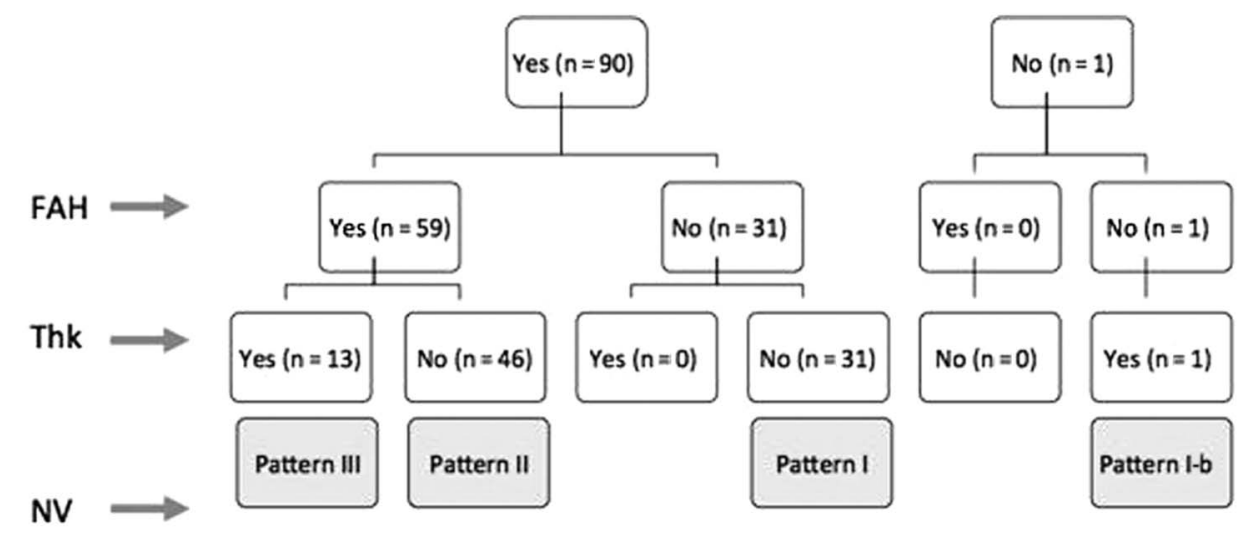

Figure 2 - Pattern combinations exhibited by the 91 tendons with abnormalities. Pattern I was found in 31 tendons; these only presented FAH abnormality. Pattern II was found in 46 tendons, presenting both FAH and Thk. Pattern III was observed in 13 tendons with FAH, Thk, and NV. Pattern I-b was detected in only 1 tendon, which displayed NV. FAH indicates focal area of hypoechogenicity; NV, neovascularisation; ThK, thickened.

Table 1 Pain Scores and Patterns of Abnormal and Normal Patellar Tendons

\begin{tabular}{|c|c|c|c|c|c|}
\hline \multirow[b]{2}{*}{ Pain measure } & \multicolumn{4}{|c|}{ Pattern observed } & \multirow[b]{2}{*}{$F$ statistic ( $P$ value) } \\
\hline & Normal $(n=55)$ & Pattern I $(n=32)$ & Pattern II $(n=46)$ & Pattern III $(n=13)$ & \\
\hline VAS & $0.6(1.2)^{\dagger}$ & $0.6(1.4) \dagger$ & $0.6(1.5)^{\dagger}$ & $3.0(3.1)^{*, * * * * * *}$ & $F_{3.142}=8.6(<.001)$ \\
\hline VISA-P & $94.6(9.1)^{\dagger}$ & $96.4(7.7)$ & $93.5(10.5)$ & $82.9(14.3) *, * *, * * *$ & $F_{3.142}=6.2(.001)$ \\
\hline PPT & $11.0(2.5)^{\dagger}$ & $10.3(3.0)$ & $10.0(2.4)$ & $8.0(2.8)^{*}$ & $F_{3.142}=4.8(.003)$ \\
\hline
\end{tabular}

Abbreviations: PPT, pain pressure threshold; VAS, visual analog scale; VISA-P, Victorian Institute of Sport assessment questionnaire-patellar tendon. Note: Measurements: VAS and VISA-P in points, PPT in $\mathrm{kg}$.

${ }^{*} P<.05$ compared with normal tendon. ${ }^{* *} P<.05$ compared with pattern I. ${ }^{* * *} P<.05$ compared with pattern II. ${ }^{\dagger} P<.05$ compared with pattern III.

A relatively low number of patterns were identified. Almost all abnormal tendons had FAH, and most exhibited Thk. Moreover, NV was found only when both structural abnormalities, FAH and Thk, were present. This finding is consistent with the continuum model of tendinopathy proposed by Cook and Purdam. ${ }^{16}$ In our experience, a patellar tendon that is subjected to a repetitive or excessive load often suffers a structural abnormality (FAH), which induces an adaptive process in which the tendon begins to thicken and, sometimes, an attempt to heal takes place, which involves the growth of NV.

The combination of the 2 structural abnormalities with NV (pattern III) obtained a worse pain score than the patterns without NV. Our findings in professional basketball players are consistent with results reported by Cook et $\mathrm{al}^{9}$ in volleyball players. Thus, our study validates Cook's results concerning the importance of the combination of structural and vascular abnormalities for explaining pain variations, while reinforcing the importance of $\mathrm{NV}$ as a mediator of patellar pain. ${ }^{17}$

Our study reveals that variations of PPT measures among abnormal tendons behave differently than the variations of the other pain measures. Thus, while the PPT did not differ between abnormal tendons with and without NV, the VAS and VISA-P scores were worse when NV was present. On the other hand, regression models evidenced that the PPT measure was the only measure determined by both NV and FAH. This different behavior between pain measures may be due to the fact that vascular and structural abnormalities have a differential effect on tendon sensitivity. Whereas NV seems to decrease the pain threshold, ${ }^{15} \mathrm{FAH}$
Table 2 Multivariate Sonographic Determinants of Each of the 3 Pain Measures

\begin{tabular}{llcc}
\hline & Abnormality & $\boldsymbol{\beta}(\mathrm{SE})$ & $\boldsymbol{P}$ value \\
\hline VAS & NV & $2.15(0.45)$ & $<.01$ \\
VISA-P & NV & $-10.5(2.78)$ & $<.01$ \\
PPT & FAH & $-0.95(0.45)$ & .04 \\
& NV & $-1.91(0.75)$ & .01 \\
\hline
\end{tabular}

Abbreviations: FAH, focal area of hypoechogenicity; NV, neovascularization; PPT, pain pressure threshold; VAS, visual analog scale; VISA-P, Victorian institute of sport assessment questionnaire-patellar tendon. Note: Presence of NV increases the VAS in 2 points. Presence of NV and FAH decreases PPT. Presence of NV decreases the VISA-P score by 10 points. Measurements: VAS and VISA-P in points, PPT in $\mathrm{kg}$.

may make tendons more sensitive to the stimulus of mechanical pressure. An application of the relevance of NV is the development of interventions aimed at reducing these abnormal vascular structures. In this sense, the evidence suggests that pain may decrease by using preventive and rehabilitation programs based on isometric exercises, ${ }^{18}$ eccentric strengthening programs for PT, ${ }^{19}$ corticosteroid injections in some cases for a short-term effect, ${ }^{20}$ load training modification, ${ }^{21}$ and other therapeutic modalities (therapeutic ultrasound, cryotherapy, transverse friction massage, bracing, or taping). ${ }^{22}$ Nevertheless, an active debate still exists regarding changes in tendon abnormalities and changes in the displayed symptoms. ${ }^{10}$ 
Most abnormal tendons without NV obtained low pain scores in all 3 measures. This may be because professional basketball players have high pain thresholds, as occurs in ultraendurance athletes, ${ }^{23}$ considering that they are bound to maximally exert themselves during their weeklong training. Nevertheless, our finding is consistent with previous research indicating that abnormal tendons can be asymptomatic. ${ }^{10,24}$ Our study adds to the existing literature by showing that the combination of 2 structural abnormalities (pattern II) does not determine differences in pain scores compared with tendons with a single, isolated structural abnormality. Previous studies have not compared pain among abnormal tendons with different numbers of structural abnormalities. ${ }^{11}$

One of the strengths of this study was the selection of adult elite basketball players from one of the most important basketball leagues, according to the European national basketball league rankings. ${ }^{25}$ Although studies on the patellar tendons of young elite or amateur basketball players are common, adult elite basketball players have received little attention.

In summary, the present study shows that the tendons exhibiting patterns of sonographic abnormalities, including NV, demonstrated greater pain. Although NV determines scores for the VAS, VISA-P, and PPT, the presence of FAH on its own is a determining factor for PPT. Classification by patterns of abnormality enables a better assessment and prognosis of the clinical status of the patellar tendon among professional basketball players. This study suggests that the combination of 2 or more sonographic abnormalities may help explain pain variations between active jumping players.

\section{Study Limitations}

Our study had several limitations. First, considering that all participants were men, caution is recommended when attempting to apply these results to women. Second, nonactive players were excluded, although their PT may have more sonographic abnormal findings and, therefore, their exclusion may bias the results regarding prevalence. Our decision to exclude these subjects was because our research question was focused on active players. Future prospective longitudinal studies are required to analyze a more representative sample, including both males and females. Likewise, future studies should compare the size of the structural abnormalities (FAH and ThK) among the different patterns identified.

\section{Perspectives}

Three main patterns or combinations of abnormalities were observed. Pattern II (which combined FAH and Thd) was the most prevalent, and pattern III (FAH, Thd, and NV) was the least common among elite basket players. NV was the only common determinant for different patellar tendon pain measures in elite players. Structural abnormalities occur prior to NV. Variations in PPT measures among abnormal tendons had a different behavior than variations in the other pain measures. We noted that the detection of tendon abnormalities in US imaging may be predictive for the development of future symptoms in patients with PT. ${ }^{11}$ Tendons with pattern I should attempt to avoid progressing to the next stage by carrying out the indicated therapeutic intervention. We observed that tendons with pattern III had significant differences and were accompanied by higher pain than other patterns.

\section{Acknowledgments}

The players volunteered their time to participate in this study. They are also grateful to the physical therapists who helped with recruitment.
The study protocol was approved by the Ethics Committee of the University of Valencia in Valencia, Spain. The authors certify that they have no affiliations with or financial involvement in any organization or entity with a direct financial interest in the subject matter or materials discussed in the article. The authors, their immediate families, and any research foundations with which they are affiliated did not receive any financial payments or other benefits from any commercial entity related to the subject of this article.

\section{References}

1. Lian OB, Engebretsen L, Bahr R. Prevalence of jumper's knee among elite athletes from different sports: a cross-sectional study. Am J Sports Med. 2005;33(4):561-567. PubMed ID: 15722279 doi:10. 1177/0363546504270454

2. Zwerver J, Bredeweg SW, van den Akker-Scheek I. Prevalence of jumper's knee among nonelite athletes from different sports: a crosssectional survey. Am J Sports Med. 2011;39:1984-1988. PubMed ID: 21737835 doi:10.1177/0363546511413370

3. Kregel J, van Wilgen P, Zwerver J. Pain assessment in patellar tendinopathy using pain pressure threshold algometry: an observational study. Pain Med. 2013;14:1769-1775. PubMed ID: 23802873 doi:10.1111/pme.12178

4. Malliaras P, Kamal B, Nowell A, et al. Patellar tendon adaptation in relation to load-intensity and contraction type. J Biomech. 2013; 46(11):1893-1899. PubMed ID: 23773532 doi:10.1016/j.jbiomech. 2013.04.022

5. Fredberg U, Bolvig L. Significance of ultrasonographically detected asymptomatic tendinosis in the patellar and Achilles tendons of elite soccer players: a longitudinal study. Am J Sports Med. 2002;30(4): 488-491. PubMed ID: 12130402 doi:10.1177/03635465020300040701

6. Khan KM, Cook JL, Kiss ZS, et al. Patellar tendon ultrasonography and jumper's knee in female basketball players: a longitudinal study. Clin J Sport Med. 1997;7(3):199-206. PubMed ID: 9262888 doi:10. 1097/00042752-199707000-00009

7. Comin J, Cook JL, Malliaras P, et al. The prevalence and clinical significance of sonographic tendon abnormalities in asymptomatic ballet dancers: a 24-month longitudinal study. Br J Sport Med. 2013; 47(2):89-92. doi:10.1136/bjsports-2012-091303

8. Khan KM, Cook JL, Bonar F, Harcourt P, Astrom M. Histopathology of common tendinopathies. Update and implications for clinical management. Sports Med. 1999;27(6):393-408. PubMed ID: 10418074 doi:10.2165/00007256-199927060-00004

9. Alfredson H, Ohberg L, Forsgren S. Is vasculo-neural ingrowth the cause of pain in chronic Achilles tendinosis? An investigation using ultrasonography and colour Doppler, immunohistochemistry, and diagnostic injections. Knee Surg Sports Traumatol Arthrosc. 2003;11(5): 334-338. PubMed ID: 14520512 doi:10.1007/s00167-003-0391-6

10. Giombini A, Dragoni S, Di Cesare A, Di Cesare M, Del Buono A, Maffulli N. Asymptomatic Achilles, patellar, and quadriceps tendinopathy: a longitudinal clinical and ultrasonographic study in elite fencers. Scand J Med Sci Sports. 2013;23(3):311-316. PubMed ID: 22092963 doi:10.1111/j.1600-0838.2011.01400.x

11. Cook JL, Malliaras P, De Luca J, Ptasznik R, Morris ME, Goldie P. Neovascularization and pain in abnormal patellar tendons of active jumping athletes. Clin J Sport Med. 2004;14(5):296-299. PubMed ID: 15377969 doi:10.1097/00042752-200409000-00008

12. Gisslén K, Alfredson H. Neovascularisation and pain in jumper's knee: a prospective clinical and sonographic study in elite junior volleyball players. Br J Sports Med. 2005;39(7):423-428. doi:10. 1136/bjsm.2004.013342 
13. Visentini PJ, Khan KM, Cook JL, Kiss ZS, Harcourt PR, Wark JD. The VISA score: an index of severity of symptoms in patients with jumper's knee (patellar tendinosis). Victorian Institute of Sport Tendon Study Group. J Sci Med Sport. 1998;1:22-28. PubMed ID: 9732118 doi:10.1016/S1440-2440(98)80005-4

14. Hernandez-Sanchez S, Hidalgo M, Gomez A. Cross-cultural adaptation of VISA-P score for patellar tendinopathy in Spanish population. J Orthop Sports Phys Ther. 2011;41:581-591. PubMed ID: 21765223 doi:10.2519/jospt.2011.3613

15. Beggs I, Bianchi S, Bueno A, et al. ESSR Ultrasound Group Protocols. Musculoskeletal Ultrasound Technical Guidelines: Knee. https://essr.org/content-essr/uploads/2016/10/knee.pdf

16. Cook JL, Purdam CR. Is tendon pathology a continuum? A pathology model to explain the clinical presentation of load-induced tendinopathy. Br J Sports Med. 2009;43(6):409-416. PubMed ID: 18812414 doi:10.1136/bjsm.2008.051193

17. Alfredson H. The chronic painful Achilles and patellar tendon: research on basic biology and treatment. Scand J Med Sci Sports. 2005;15:252-259. PubMed ID: 15998342 doi:10.1111/j.1600-0838. 2005.00466.x

18. Rio E, Kidgell D, Purdam C, et al. Isometric exercise induces analgesia and reduces inhibition in patellar tendinopathy. Br J Sports Med. 2015;49(19):1277-1283. PubMed ID: 25979840 doi:10.1136/ bjsports-2014-094386

19. Purdam CR, Jonsson P, Alfredson H, Lorentzon R, Cook JL, Khan $\mathrm{KM}$. A pilot study of the eccentric decline squat in the management of painful chronic patellar tendinopathy. Br J Sports Med. 2004; 38(4):395-397. PubMed ID: 15273169 doi:10.1136/bjsm.2003. 000053

20. Kongsgaard M, Kovanen V, Aagaard P, et al. Corticosteroid injections, eccentric decline squat training and heavy slow resistance training in patellar tendinopathy. Scand J Med Sci Sports. 2009; 19(6):790-802. PubMed ID: 19793213 doi:10.1111/j.1600-0838. 2009.00949.x

21. Cook JL, Khan KM, Purdam CR. Conservative treatment of patellar tendinopathy. Phys Ther Sport. 2001;2(2):54-65. doi:10.1054/ptsp. 2001.0069

22. Reinking MF. Current concepts in the treatment of patellar tendinopathy. Int J Sports Phys Ther. 2016;11(6):854-866. PubMed ID: 27904789

23. Freund W, Weber F, Billich C, Birklein F, Breimhorst M, Schuetz UH. Ultra-marathon runners are different: investigations into pain tolerance and personality traits of participants of the TransEurope FootRace 2009. Pain Pract. 2013;13:524-532. PubMed ID: 23368760 doi:10.1111/papr.12039

24. Robinson JM, Cook JL, Purdam C, et al. The VISA-A questionnaire: a valid and reliable index of the clinical severity of Achilles tendinopathy. Br J Sports Med. 2001;35(5):335-341. PubMed ID: 11579069 doi:10.1136/bjsm.35.5.335

25. Country Ranking (1950-2014). FIBA World Cup Based. https:// gigabasket.org. Accessed July 1, 2016. 